\title{
7. Pathological and Clinical Characteristics of the Proposed New Entities
}

Makek M: Clinical Pathology of Fibro-Osteo-Cemental Lesions in the Cranio-Facial and Jaw Bones. Basel, Karger, 1983, pp 105-211 